\title{
Baseline measure of health-related quality of life (Functional Assessment of Cancer Therapy-Esophagus) is associated with overall survival in patients with esophageal cancer
}

\author{
Biniam Kidane, MD, MSc, ${ }^{\text {a,b }}$ Joanne Sulman, MSW, ${ }^{\text {,c }}$ Wei Xu, PhD, ${ }^{\mathrm{d}}$ Qin Quinn Kong, MSc, ${ }^{\mathrm{d}}$ \\ Rebecca Wong, MBChB, MSc, ,e Jennifer J. Knox, MD, MSc, d,f and Gail E. Darling, MD ${ }^{\mathrm{a}, \mathrm{b}, \mathrm{d}}$
}

\begin{abstract}
Objective: Functional Assessment of Cancer Therapy-Esophagus is a healthrelated quality of life instrument validated in patients with esophageal cancer. It is composed of a general component and an esophageal cancer subscale. Our objective was to determine whether the baseline Functional Assessment of Cancer Therapy-Esophagus and esophageal cancer subscale scores are associated with survival in patients with stage II and III cancer of the gastroesophageal junction or thoracic esophagus.
\end{abstract}

Methods: Data from 4 prospective studies in Canadian academic hospitals were combined. These included consecutive patients with stage II and III esophageal cancer who received neoadjuvant therapy followed by surgery or chemoradiation/radiation alone. All patients completed baseline Functional Assessment of Cancer Therapy-Esophagus. Functional Assessment of Cancer TherapyEsophagus and esophageal cancer subscale scores were dichotomized on the basis of median scores. Cox regression analyses were performed.

Results: There were 207 patients treated between 1996 and 2014. Mean age was $61 \pm 10.6$ years. Approximately $69.6 \%$ of patients $(n=144)$ had adenocarcinoma. All patients had more than 9 months of follow-up. In patients with stage II and III, 93 deaths were observed. When treated as continuous variables, baseline Functional Assessment of Cancer Therapy-Esophagus and esophageal cancer subscale were associated with survival with hazard ratios of $0.89(95 \%$ confidence interval [CI], 0.81-0.96; $P=.005)$ and 0.68 (95\% CI, 0.56-0.82; $P<.001)$, respectively. When dichotomized, they were also associated with survival with a hazard ratio of $0.58(95 \% \mathrm{CI}, 0.38-0.89 ; P=.01)$ and 0.43 (95\% CI, 0.28-0.67; $P<.001)$, respectively.

Conclusions: In patients with stage II and III esophageal cancer being considered for therapy, higher baseline Functional Assessment of Cancer TherapyEsophagus and esophageal cancer subscale were independently associated with longer survival, even after adjusting for age, stage, histology, and therapy received. Further study is needed, but Functional Assessment of Cancer Therapy-Esophagus may be useful as a prognostic tool to inform patient decision-making and patient selection criteria for studies. (J Thorac Cardiovasc Surg 2016;151:1571-80)

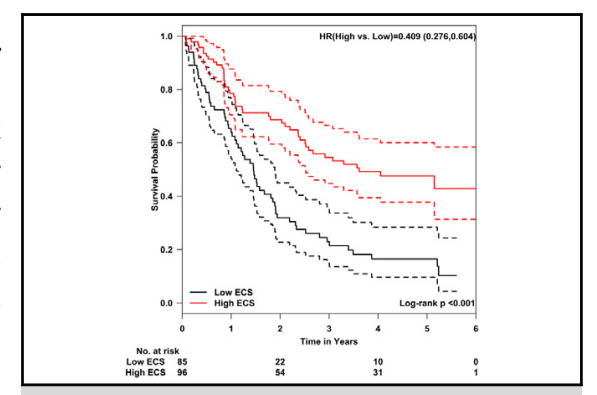

Lower baseline HRQOL (FACT-E) is associated with poorer survival.

\section{Central Message}

In patients with stage II and III esophageal cancer, lower HRQOL before therapy is associated with worse survival.

\section{Perspective}

In patients with stage II and III esophagea cancer before therapy, higher baseline HRQOL (FACT-E) is associated with higher overall survival. This association persists even after controlling for stage and treatment. Further study is needed, but FACT-E may be a useful prognostic tool to inform patient decisionmaking and selection criteria for studies.

See Editorial page 1448.

\footnotetext{
From the ${ }^{\mathrm{a}}$ Division of Thoracic Surgery, ${ }^{\mathrm{c}}$ Factor-Inwentash Faculty of Social Work, ${ }^{\mathrm{e}}$ Department of Radiation Oncology, and ${ }^{\mathrm{f}}$ Department of Medical Oncology, University of Toronto; ${ }^{b}$ Divison of Thoracic Surgery, Toronto General Hospital, and ${ }^{\mathrm{d}}$ Princess Margaret Cancer Center, University Health Network, Toronto, Ontario, Canada.

Read at the 95th Annual Meeting of The American Association for Thoracic Sur-
} gery, Seattle, Washington, April 25-29, 2015.
Received for publication April 28, 2015; revisions received Dec 29, 2015; accepted for publication Jan 17, 2016; available ahead of print March 23, 2016.

Address for reprints: Gail E. Darling, MD, Division of Thoracic Surgery, Toronto General Hospital, University Health Network, 200 Elizabeth St 9N-955, Toronto, Ontario, Canada M5G 2C4 (E-mail: gail.darling@uhn.on.ca). $0022-5223 / \$ 36.00$

Copyright (c) 2016 by The American Association for Thoracic Surgery http://dx.doi.org/10.1016/j.jtcvs.2016.01.052 


$$
\begin{aligned}
& \text { Abbreviations and Acronyms } \\
& \text { CI }=\text { confidence interval } \\
& \text { ECS }=\text { esophageal cancer subscale } \\
& \text { EORTC = European Organization for Research } \\
& \text { and Treatment of Cancer } \\
& \text { FACT-E = } \text { Functional Assessment of Cancer } \\
& \text { Therapy-Esophagus } \\
& \text { FACT-G = } \text { Functional Assessment of Cancer } \\
& \text { Therapy-General } \\
& \text { HR }= \text { hazard ratio } \\
& \text { HRQOL }= \text { health-related quality of life } \\
& \text { QLQ }= \text { Quality of Life Questionnaire }
\end{aligned}
$$

\section{Scanning this QR code will take you to the article title page. To view the AATS 2015 Webcast, see the URL at the end of the article.}

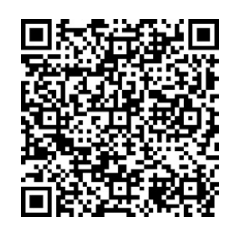

Esophageal cancer has a significant effect on health-related quality of life (HRQOL). ${ }^{1}$ Dysphagia is the primary symptom leading the patient to seek medical attention. In addition to the physical problem of difficulty swallowing or the weight loss that may result, social isolation may occur because the inability to swallow comfortably or the risk of food regurgitation may lead to increasing withdrawal from social situations. Poor HRQOL has been shown to be associated with poorer long-term survival in several cancers, ${ }^{1-7}$ primarily in those with lung and breast cancer, but there is also evidence demonstrating that poor HRQOL is associated with poorer long-term survival in patients with esophageal cancer. ${ }^{2,3,5,6}$ However, these studies were based on post-treatment HRQOL or changes of HRQOL over the course of treatment. ${ }^{2,3,5,6}$ Given that esophageal cancer initially may present with significant HRQOL issues, it seems likely that baseline HRQOL may be an especially important prognostic tool in this patient population.

The Functional Assessment of Cancer TherapyEsophagus (FACT-E) is an HRQOL instrument validated in patients with esophageal cancer. It is composed of a general component (FACT-G) and an esophageal cancer subscale (ECS). Our objective was to determine whether the baseline FACT-E or the baseline ECS alone is associated with overall survival in patients with stage II and III cancer of the gastroesophageal junction or thoracic esophagus.

\section{MATERIALS AND METHODS \\ Patient Population}

Data from 4 prospective, nonrandomized studies in 3 large Canadian academic hospitals (Toronto General Hospital, Princess Margaret Cancer
Centre, The Ottawa Hospital) were combined. Table 1 provides a brief description of these studies. The current study and all 4 studies were approved by institutional ethics boards. ${ }^{8,9}$ These studies included consecutive eligible patients with clinical stage II or IV cancer of the gastroesophageal junction or thoracic esophagus who received chemotherapy (mostly with cisplatin and irinotecan for up to 3 cycles) and concomitant radiation (typically 50 Gy in 25 fractions) as neoadjuvant therapy or as part of bimodality therapy without surgery. For the purposes of this current study, patients were classified as having received chemotherapy or radiation therapy if they completed at least $50 \%$ of the intended therapy. In 1 of the 4 studies, adjuvant sunitinib was used. Sunitinib is a broad-spectrum orally available multitargeted tyrosine kinase inhibitor with antiangiogenic and antitumor activity. ${ }^{10}$ For the purposes of this current study, patients were classified as having used adjuvant sunitinib if they had at least 6 months of sunitinib therapy.

The surgical techniques used included McKeown esophagectomy, Ivor-Lewis resection, left thoracoabdominal approach with a left neck incision, minimally invasive esophagectomy, and transhiatal esophagectomy. En bloc resection of overlying mediastinal pleura, a cuff of the diaphragmatic crura, and mediastinal fat was recommended. Recommended lymphadenectomy included lower mediastinal and abdominal lymph nodes. Proximal and distal margins, measured in the operating room, were more than $5 \mathrm{~cm}$ beyond the macroscopic tumor. Clinical follow-up after surgery or definitive chemoradiation therapy occurred every 3 months until 2 years postoperatively followed by every 6 months until 5 years postoperatively. Radiologic follow-up occurred with at least yearly computed tomography or chest x-ray for 5 years. Staging was defined according to the 7th edition of the American Joint Committee on Cancer TNM staging system. Pathologic stage was used to describe those who underwent surgery, and clinical stage was used to describe those who did not undergo surgery.

\section{Health-Related Quality of Life Instrument}

The FACT-G is a general HRQOL instrument that has been validated for use in any cancer; it consists of 28 questions covering the domains of physical well-being, functional well-being, social well-being, and emotional well-being using a 5-point Likert scale. ${ }^{9}$ A disease-specific module, the ECS was validated and added to the FACT-G to result in the FACT-E for esophageal cancer. ${ }^{9}$ The ECS consists of 17 items addressing eating, swallowing, enjoyment of food, voice, dry mouth, appetite, cough, choking, and pain; each of these is evaluated using a 5-point Likert scale to generate a summary score of esophageal-specific concerns. ${ }^{9}$ Higher scores denote better quality of life. All patients completed FACT-E at baseline; surveys were self-administered at the time of the first consultation with the thoracic surgeon or medical or radiation oncologist before initiation of any therapies.

\section{Statistical Analysis}

For univariate analysis, normally distributed continuous data were reported as means with standard deviations and analyzed using independent sample $t$ tests. Data that were not normally distributed were reported as medians with interquartile ranges and analyzed using the Mann-Whitney $U$ test. Fisher exact tests were used for univariate analysis of categoric data. Multivariate time-to-event analyses were performed using Cox regression analyses. FACT-E and ECS were our main predictors of interest. We performed separate Cox regression analyses including only 1 HRQOL for each model (ECS or FACT-E). A forced-entry method was used to control for the following variables: age, stage, histology (adenocarcinoma vs nonadenocarcinoma), year of treatment, and whether the patient received surgery, chemotherapy, radiation, or adjuvant sunitinib therapy. The variable "curative intent" was used to distinguish those patients who received chemoradiation therapy as a curative modality (ie, in select patients with squamous cell cancer) from those who received it on a palliative basis (ie, unresectable or metastatic disease). The FACT-E 
TABLE 1. Description of component studies

\begin{tabular}{|c|c|c|c|c|}
\hline & Study 1 & Study 2 & Study 3 & Study 4 \\
\hline Sample size contribution & 45 & 60 & 53 & 49 \\
\hline \multirow[t]{4}{*}{ Inclusion criteria } & & 1. Clinical stage $2-4$ & & 1. Clinical stage $2-3$ \\
\hline & & 2. ECOG 0-2 & & 2. ECOG $0-2$ \\
\hline & & 3. English-speaking & & 3. English-speaking \\
\hline & \multicolumn{3}{|c|}{ 4. Competent to provide informed consent } & 4. Able to provide informed consent \\
\hline Study design & Single-arm phase 2 & Prospective cohort & Prospective cohort & Single-arm phase 2 \\
\hline Time period & $2002-2005$ & $1996-2002$ & 2012-2014 & $2006-2010$ \\
\hline Publication status & Published $^{8}$ & Published $^{9}$ & Not yet published & Accepted for publication \\
\hline
\end{tabular}

ECOG, Eastern Cooperative Oncology Group.

and ECS scores were dichotomized to high and low HRQOL groups based on the median FACT-E and ECS scores. Cox regression analyses were performed treating FACT-E and ECS as both continuous and dichotomous variables. Further sensitivity analyses also were performed with FACT-E and ECS quartiles rather than as dichotomous or continuous. Because the primary analyses excluded patients with stage IV, we then subsequently performed sensitivity analyses including patients with stage IV. For further sensitivity analyses, we also performed the univariate and Cox regression analyses using clinical stage uniformly for all patients. Hazard ratios (HRs) and associated Kaplan-Meier curves were produced. When interpreting the results of Cox regression analyses treating FACT-E and ECS as continuous variables, the HR represents the increase or decrease in survival associated with a 10-point decrease in FACT-E or ECS score.

As a preliminary test of prognostic ability, 2 multivariate logistic regression models were constructed with the outcome being "survival for more than 2 years" for both models. This outcome was chosen because it was a clinically relevant period of survival that would warrant aggressive therapy. The main predictor of interest in 1 model was baseline FACT-E; this model did not include ECS as a stand-alone variable. Baseline stand-alone ECS was the main predictor of interest in the other model; this model did not include the overall FACT-E. The purpose of these models was to assess whether they could predict 2-year survivors using only data available at presentation (before any treatments). Thus, the other covariates in the models were age, clinical stage, and histology (adenocarcinoma vs nonadenocarcinoma).

A 2-sided alpha of 0.05 was used for all tests of significance. Statistical analyses were performed using the SAS statistical package (version 9.3; SAS Institute, Inc, Cary, NC).

\section{RESULTS}

There were 207 patients with stage II and IV esophageal cancer treated between 1996 and 2014; 194 patients $(93.7 \%)$ were treated at the University Health Network (Toronto General Hospital and Princess Margaret Cancer Centre), and 13 patients were treated at the Ottawa Hospital. Thirty-five patients had stage IV disease and were treated with palliative intent, whereas the remaining 172 patients with stage II or III disease were treated with curative intent (Table 2). The completion rate of baseline FACT-E survey was $100 \%$. Mean age was $61 \pm 10.6$ years, and 144 patients $(69.6 \%)$ had adenocarcinoma. The distribution of FACT-E and ECS scores is shown in Figure 1.

The majority of patients $(62.3 \%, \mathrm{n}=129)$ received neoadjuvant chemoradiation or perioperative chemotherapy (preoperative and postoperative). An additional 25 patients $(12.1 \%)$ received surgery only, and the majority of the remaining patients received definitive or palliative chemoradiation therapy. A few also received just chemotherapy or radiation therapy because of an inability to tolerate combined therapy. Fourteen patients $(6.8 \%)$ completed 6 to 12 months of therapy with adjuvant sunitinib. The distribution of surgical approaches was as follows: McKeown esophagectomy $(\mathrm{n}=27,17 \%)$, Ivor-Lewis resection $(\mathrm{n}=49,31 \%)$, left thoracoabdominal approach with a left neck incision $(\mathrm{n}=53,34 \%)$, and transhiatal esophagectomy $(\mathrm{n}=17,11 \%)$. Minimally invasive esophagectomy, most commonly a minimally invasive Ivor-Lewis, was performed in 11 patients $(7 \%)$.

With more than 9 months of follow-up for all patients, 114 deaths were observed. By excluding patients in stage IV, 93 deaths were observed in the patients treated with curative intent. Mean and median follow-up were 2.28 and 1.78 years, respectively. On unadjusted/univariate analysis, higher mean FACT-E and ECS were associated with overall survival (Table 3). On multivariate/adjusted

TABLE 2. Description of population

\begin{tabular}{lc}
\hline \multicolumn{1}{c}{ Variable } & Number \\
\hline Mean age (SD) & $61.0(10.6)$ \\
Adenocarcinoma, N (\%) & $144(69.6 \%)$ \\
Stage & \\
2 & $81(39.1 \%)$ \\
3 & $91(44.0 \%)$ \\
4 & $35(16.9 \%)$ \\
Received chemotherapy, N (\%) & $159(76.8 \%)$ \\
Received cisplatin + irinotecan, N (\%) & $148(71.5 \%)$ \\
Received cisplatin + 5-FU, N (\%) & $8(3.9 \%)$ \\
Received cisplatin + 5-FU + etoposide, N (\%) & $3(1.4 \%)$ \\
Received radiation therapy, N (\%) & $148(75.1 \%)$ \\
Surgery, N (\%) & $157(75.8 \%)$ \\
Received adjuvant sunitinib, N (\%) & $14(6.8 \%)$ \\
Received chemoradiation + surgery, N (\%) & $108(52.2 \%)$ \\
Received chemotherapy + surgery, N (\%) & $21(10.1 \%)$ \\
Received surgery only, N (\%) & $25(12.1 \%)$ \\
Curative intent therapy, N (\%) & $172(83.1 \%)$ \\
Mortality, N (\%) & $114(55.1 \%)$ \\
Mean FACT-E (SD) & $78.2(17.5)$ \\
Mean ECS (SD) & $45.4(12.9)$ \\
\hline SD, Standard deviation; 5-FU, 5-fluorouracil; FACTE Function
\end{tabular}

$S D$, Standard deviation; 5-FU, 5-fluorouracil; FACT-E, Functional Assessment of Cancer Therapy-Esophagus; $E C S$, esophageal cancer subscale. 

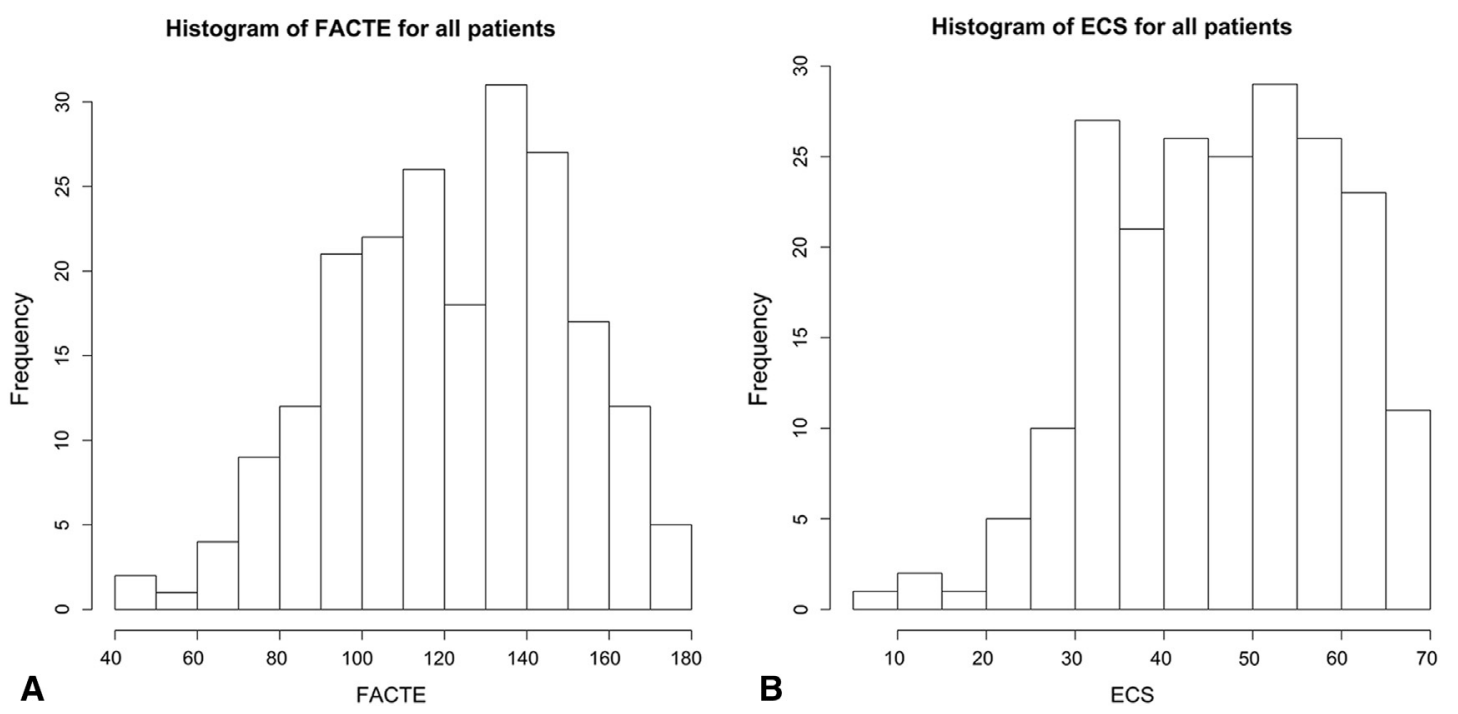

FIGURE 1. Histogram of FACT-E and ECS distributions. Histogram depicting the distribution of (A) FACT-E scores and (B) ECS scores. FACT-E, Functional Assessment of Cancer Therapy-Esophagus; ECS, esophageal cancer subscale.

Cox regression analysis controlling for the confounding effects of age, stage, histology (adenocarcinoma vs nonadenocarcinoma), year of treatment, and whether the patient received surgery, chemotherapy, radiation, or adjuvant sunitinib therapy, both baseline FACT-E and ECS were independently associated with overall survival. When treated as continuous variables, lower baseline FACT-E and ECS were significantly associated with worse overall survival with HRs of 0.89 (95\% confidence interval $[\mathrm{CI}], 0.81-0.96 ; P=.005)$ and 0.68 (95\% CI, 0.56-0.82; $P<.001)$, respectively. When treated as dichotomous variables, lower baseline FACT-E and ECS were significantly associated with worse overall survival with HRs of $0.53(95 \% \mathrm{CI}, 0.33-0.82 ; P=.005)$ and 0.46

TABLE 3. Univariate predictors of overall survival (excluding patients in stage IV)

\begin{tabular}{lccc}
\hline \multicolumn{1}{c}{ Predictor } & $\begin{array}{c}\text { Alive } \\
(\mathbf{n = 7 9 )}\end{array}$ & $\begin{array}{c}\text { Mortality } \\
(\mathbf{n = 9 3 )}\end{array}$ & $\boldsymbol{P}$ value \\
\hline Mean age (SD), y & $60.6(11.5)$ & $62.0(9.4)$ & .38 \\
Adenocarcinoma, N (\%) & $56(70.9 \%)$ & $67(72.0 \%)$ & .87 \\
Stage & & & .28 \\
2 & $41(51.9 \%)$ & $40(43.0 \%)$ & \\
3 & $38(48.1 \%)$ & $53(57.0 \%)$ & \\
Received chemotherapy, & $64(81.0 \%)$ & $74(79.6 \%)$ & .85 \\
$\quad$ N (\%) & & & \\
Received radiation therapy, & $60(75.9 \%)$ & $65(69.9 \%)$ & .40 \\
$\quad$ N (\%) & $71(89.9 \%)$ & $81(87.1 \%)$ & .32 \\
Surgery, N (\%) & $9(11.4 \%)$ & $5(5.4 \%)$ & .17 \\
Received adjuvant sunitinib, & & & \\
$\quad$ N (\%) & $79.0(18.2)$ & $74.3(18.5)$ & $\mathbf{. 0 5}$ \\
Mean FACT-E (SD) & $50.2(12.4)$ & $44.2(12.2)$ & $\mathbf{. 0 0 2}$ \\
Mean ECS (SD)
\end{tabular}

$S D$, Standard deviation; FACT-E, Functional Assessment of Cancer TherapyEsophagus; ECS, esophageal cancer subscale. Bold signifies statistically significant result.
(95\% CI, 0.29-0.72; $P<.001$ ), respectively (Figure 2). Tables 4 and 5 show the results of adjusted Cox regression analyses using FACT-E (Table 4) and ECS (Table 5) as dichotomous variables. The C-statistics for the FACT-E model and the ECS model were 0.71 (95\% CI, 0.65-0.76) and 0.72 (95\% CI, 0.66-0.78), respectively. Sensitivity analysis considering baseline FACT-E and ECS as quartiles showed a significant trend of increasing survival with increasing baseline FACT-E $(P=.02)$ and ECS $(P<.001)$ quartile (Figure 3$)$. Sensitivity analyses using clinical stage rather than pathologic stage replicated the findings that FACT-E and ECS were independently associated with survival and that stage did not appear to be independently and significantly associated with survival in this cohort. Sensitivity analyses including patients with stage 4 also replicated these findings.

Table 6 shows the results of a multivariable logistic regression for predictors of 2-year survival using only variables known at baseline before any treatment. Addition of FACT-E to the reduced model had the following effects: (1) changed the model from being nonpredictive to being predictive of 2-year survival (Omnibus test $P=.54$ to $P=.03$ ); (2) increased the pseudoR ${ }^{2}$ by 0.04 ; and (3) increased the C-statistic by 0.11. Addition of ECS to the reduced model had the following effects: (1) changed the model from being nonpredictive to being predictive of 2-year survival (Omnibus test $P=.54$ to $P<.001$ ); (2) increased the pseudo ${ }^{2}$ by 0.21 ; and (3) increased the $\mathrm{C}$-statistic by 0.10 . Thus, these analyses suggest that FACT-E or ECS, when added to routine clinical data, improves the ability of the model to predict 2-year overall survival. Moreover, it appears that FACT-E and ECS are the predictors that drive the ability of these particular models (including age, stage, histology, and performance 


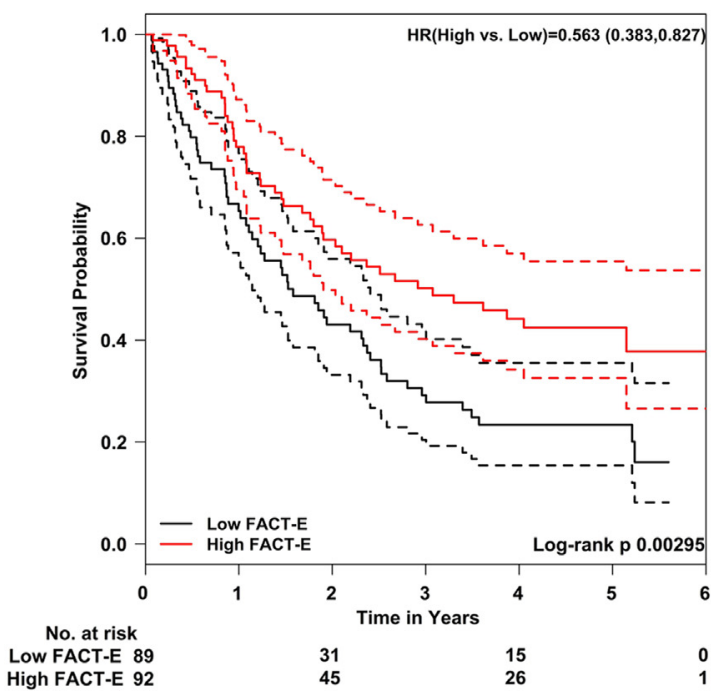

A

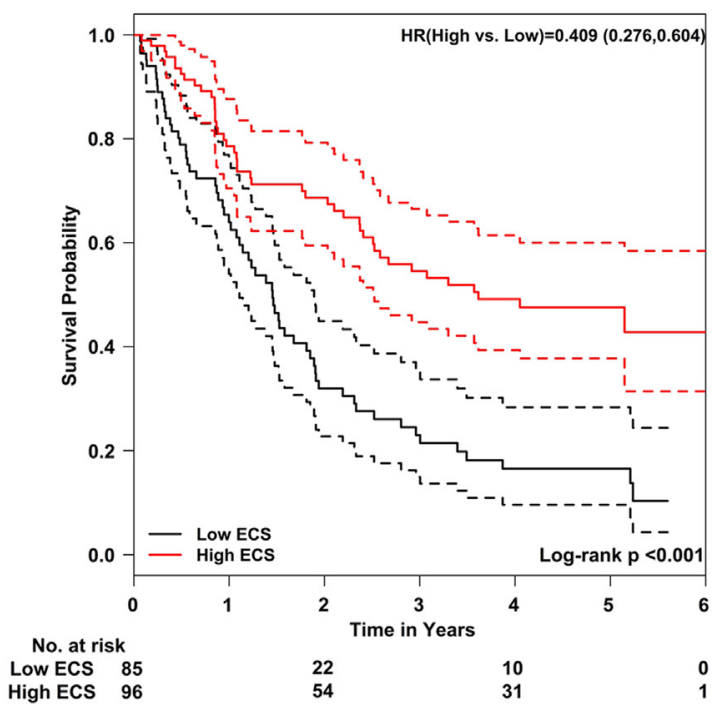

B baseline FACT-E stratified into low and high FACT-E groups. B, Kaplan-Meier curve showing results of Cox regression analysis with baseline ECS stratified into low and high ECS groups. Solid lines represent the observed survival curves, and dotted lines represent the 95\% CIs. Low baseline FACT-E and ECS are associated with worse overall survival. HR, Hazard ratio; FACT-E, Functional Assessment of Cancer Therapy-Esophagus; ECS, esophageal cancer subscale.

status) to predict 2-year overall survival. Furthermore, these analyses suggest that ECS is a stronger predictor of 2-year overall survival than FACT-E.

\section{DISCUSSION}

In patients with stage II and III esophageal cancer being considered for curative intent therapy, higher baseline FACT-E or higher baseline ECS was independently associated with better overall survival. Previous studies reporting an association of HRQOL and survival have used post-treatment HRQOL or change in post-treatment

TABLE 4. Multivariate Cox regression analysis for overall survival using Functional Assessment of Cancer Therapy-Esophagus as health-related quality of live variable (excluding patients in stage IV)

\begin{tabular}{lcc}
\hline \multicolumn{1}{c}{ Predictor } & HR $(\mathbf{9 5} \% \mathbf{C I})$ & $\boldsymbol{P}$ value \\
\hline Age (HR for 1-y increase in age) & $1.02(0.99-1.04)$ & .08 \\
Squamous cell histology & $0.72(0.44-1.16)$ & .18 \\
Stage (II vs III) & $1.23(0.78-1.94)$ & .38 \\
Received chemotherapy & $0.44(0.21-0.92)$ & $\mathbf{. 0 3}$ \\
Received radiation therapy & $1.37(0.73-2.6)$ & .33 \\
Received adjuvant sunitinib & $0.37(0.14-0.99)$ & .05 \\
Surgery & $0.58(0.28-1.21)$ & .15 \\
Year of treatment & & $\mathbf{. 0 2}$ \\
$\quad$ 1995-1999 & Reference & \\
2000-2004 & $0.52(0.24-1.15)$ & \\
2005-2009 & $1.38(0.63-3.04)$ & \\
2010-2014 & $1.68(0.63-4.45)$ & \\
FACT-E (HR for 10-point increase) & $0.52(0.33-0.82)$ & $\mathbf{. 0 0 5}$ \\
\hline
\end{tabular}

$H R$, Hazard ratio; $C I$, confidence interval; FACT-E, Functional Assessment of Cancer Therapy-Esophagus. Bold signifies statistically significant result.
HRQOL. The additional ability of baseline HRQOL to discriminate patient outcomes, when added to models using stage and type of therapy, may allow the development of a prognostic model to guide clinicians and patients in treatment decision-making. The ECS appears to have an even stronger association than FACT-E, as demonstrated by its HR and tighter CIs. Furthermore, ECS is a focused, shorter questionnaire. ' However, use of the ECS independently requires further study because it has not been validated for use independent of the FACT-E. Our findings also suggest that the addition of FACT-E alone or ECS alone to statistical models will enhance the ability

TABLE 5. Multivariate Cox regression analysis for overall survival using esophageal cancer subscale as health-related quality of life variable (excluding patients in stage IV)

\begin{tabular}{lcc}
\hline \multicolumn{1}{c}{ Predictor } & HR $(\mathbf{9 5} \% \mathbf{C I})$ & $\boldsymbol{P}$ value \\
\hline Age (HR for 1-y increase in age) & $1.02(0.99-1.04)$ & .13 \\
Squamous cell histology & $0.78(0.48-1.27)$ & .18 \\
Stage (II vs III) & $1.36(0.87-2.14)$ & .31 \\
Received chemotherapy & $0.47(0.23-0.98)$ & $\mathbf{. 0 4}$ \\
Received radiation therapy & $1.54(0.81-2.95)$ & .19 \\
Received adjuvant sunitinib & $0.43(0.16-1.17)$ & .10 \\
Surgery & $0.54(0.26-1.13)$ & .10 \\
Year of treatment & & $\mathbf{. 0 3}$ \\
$\quad$ 1995-1999 & Reference & \\
2000-2004 & $0.60(0.27-1.31)$ & \\
2005-2009 & $1.36(0.62-2.98)$ & \\
2010-2014 & $1.36(0.53-3.48)$ & \\
ECS (HR for 10-point increase) & $0.46(0.29-0.72)$ & $\mathbf{. 0 0 1}$ \\
\hline
\end{tabular}

$H R$, Hazard ratio; $C I$, confidence interval; $E C S$, esophageal cancer subscale. Bold signifies statistically significant result. 

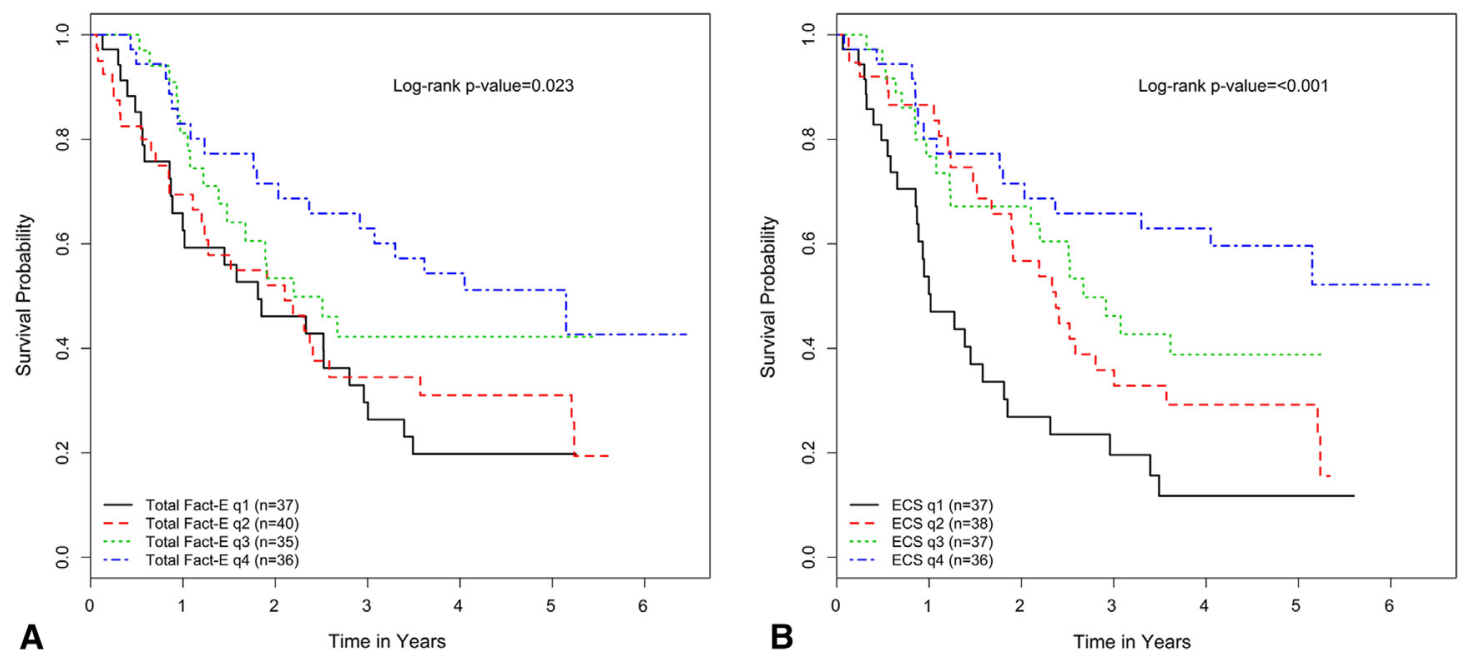

FIGURE 3. Increasing FACT-E and ECS quartiles associated with increasing overall survival. Kaplan-Meier curve showing results of Cox regression analysis with baseline (A) FACT-E and (B) ECS stratified into quartile groups. Numbering of quartiles is such that HRQOL is increasing as quartile number increases (ie, quartile 1 has lowest HRQOL). Increasing FACT-E and ECS quartiles are associated with increasing overall survival. FACT-E, Functional Assessment of Cancer Therapy-Esophagus; ECS, esophageal cancer subscale.

to predict 2-year survival in analyses that consider only variables available before treatment. It also appears that these HRQOL measures are the predictors that drive the ability of these particular models (including stage and histology) to predict 2-year survival. Furthermore, our findings suggest that ECS is a stronger predictor of 2-year overall survival than FACT-E.

In the esophageal cancer literature, it has been shown that poor HRQOL is associated with poorer long-term survival in patients with esophageal cancer. ${ }^{2,3,5-7,11,12}$ In contrast to our study, the predictor of survival in these studies was posttreatment HRQOL or changes of HRQOL over the course of treatment. ${ }^{2,3,5,6}$ Thus, this required patients to have undergone some therapy, and the effect of that therapy itself may have modified the HRQOL measurements. ${ }^{2,3,5,6}$ Although the association between survival and changes in HRQOL in response to therapy is a valuable addition to the literature, prognostication would be improved if HRQOL measured before any initiation of therapy was predictive of overall survival. The HRQOL instrument used in the majority of studies was the European Organization for Research and Treatment of Cancer Quality of Life Questionnaire-30 (EORTC QLQ-30) with the esophageal cancer disease-specific module esophagus 18.,.$^{3,5-7,11}$ By using a 4-point Likert scale, the QLQ-30 includes 5 functional scales (physical, emotional, social, cognitive, and role function) and 3 symptom scales (fatigue, nausea, and pain). ${ }^{1,13}$ There are 6 single-item questions (dyspnea, insomnia, appetite, constipation, diarrhea, and financial worries) that are also evaluated using a 4-point Likert scale. ${ }^{1,13}$ Global QOL is assessed by a single question using a 7-point Likert scale. ${ }^{1,13}$ The esophageal module (esophagus 18) includes 18 items addressing symptoms specific to esophageal cancer (eg, dysphagia). ${ }^{1,13}$ The studies that have used FACT-E as the HRQOL instrument have tended to be North American studies. ${ }^{2,12}$

Although some studies have explored whether baseline HRQOL can predict long-term survival in other cancer types, studies in esophageal cancer are more limited. . $^{3,4,7,11,14,15}$ The few studies that have explored this matter in patients with esophageal cancer all have been in European patient populations. ${ }^{7,11,15}$ Blazeby and colleagues $^{7}$ first showed that baseline HRQOL using the EORTC QLQ-30 was associated with survival in a study conducted in an era (1993-1995) before the trials showing benefit for neoadjuvant and perioperative therapy. In their study of 89 patients with stage II to IV esophageal cancer (of whom only 38 were alive at 6 months post-therapy), they showed that physical function was independently associated with overall survival. ${ }^{7}$ However, a limitation of this study was that they did not control for many important confounders, such as type of therapy given (ie, surgery, chemotherapy, radiation therapy). Moreover, this study's results, although groundbreaking, are not as applicable today in the era of better staging methodology and use of neoadjuvant and perioperative therapy. The fact that only $43 \%$ (38/89) of patients were alive at 6 months post-therapy supports this position. ${ }^{7}$ More recently, van Heijl and colleagues ${ }^{11}$ reported on 199 patients from 2 centers, of whom 187 completed baseline HRQOL instruments. They limited their study to patients with adenocarcinoma and who received only surgical therapy. ${ }^{11}$ They found that the physical symptoms component of the HRQOL instrument was independently associated with overall survival. ${ }^{11}$ However, a limitation of this study is that they used an unvalidated modification of the Rotterdam Symptom 
TABLE 6. Multivariate logistic regression model for predictors of 2-year survival

\begin{tabular}{|c|c|c|c|c|c|}
\hline \multicolumn{3}{|c|}{ Model 1: FACT-E } & \multicolumn{3}{|c|}{ Model 2: ECS } \\
\hline \multicolumn{2}{|c|}{ Reduced model (without FACT-E) } & \multirow{2}{*}{$\frac{P \text { value }}{.20}$} & \multicolumn{2}{|c|}{ Reduced model (without ECS) } & \multirow{2}{*}{$\frac{P \text { value }}{.20}$} \\
\hline Adenocarcinoma & $\begin{array}{c}\text { OR, } 0.60 \\
(95 \% \text { CI, } 0.28-1.31)\end{array}$ & & Adenocarcinoma & $\begin{array}{c}\text { OR, } 0.60 \\
(95 \% \text { CI, } 0.28-1.31)\end{array}$ & \\
\hline Clinical stage & $\begin{array}{c}\text { OR, } 0.70 \\
(95 \% \text { CI, } 0.40-1.84)\end{array}$ & .70 & Clinical stage & $\begin{array}{c}\text { OR, } 0.70 \\
(95 \% \text { CI, } 0.40-1.84)\end{array}$ & .70 \\
\hline Age & $\begin{array}{c}\text { OR, } 0.98 \\
(95 \% \text { CI, } 0.94-1.02)\end{array}$ & .24 & Age & $\begin{array}{c}\text { OR, } 0.98 \\
(95 \% \text { CI, } 0.94-1.02)\end{array}$ & .24 \\
\hline ECOG performance status & $\begin{array}{c}\text { OR, } 1.06 \\
(95 \% \text { CI, } 0.50-1.84)\end{array}$ & .87 & ECOG performance status & $\begin{array}{c}\text { OR, } 1.06 \\
(95 \% \text { CI, } 0.50-1.84)\end{array}$ & .87 \\
\hline Omnibus test of model coefficients & N/A & .54 & Omnibus test of model coefficients & N/A & .54 \\
\hline Hosmer-Lemeshow test & N/A & .78 & Hosmer-Lemeshow test & N/A & .78 \\
\hline Nagelkerke pseudoR $^{2}$ & 0.03 & N/A & Nagelkerke pseudoR ${ }^{2}$ & 0.03 & N/A \\
\hline C-statistic & 0.60 & N/A & C-statistic & 0.60 & N/A \\
\hline \multicolumn{2}{|c|}{ Full model (with FACT-E) } & $P$ value & \multicolumn{2}{|c|}{ Full model (with ECS) } & $P$ value \\
\hline FACT-E & $\begin{array}{c}\text { OR, } 1.02 \\
(95 \% \text { CI, } 1.01-1.04)\end{array}$ & .005 & ECS & $\begin{array}{c}\text { OR, } 1.06 \\
(95 \% \text { CI, } 1.04-1.10)\end{array}$ & $<.001$ \\
\hline Adenocarcinoma & $\begin{array}{c}\text { OR, } 0.44 \\
(95 \% \text { CI, } 0.19-1.07)\end{array}$ & .06 & Adenocarcinoma & $\begin{array}{c}\text { OR, } 0.41 \\
(95 \% \text { CI, } 0.17-1.00)\end{array}$ & .05 \\
\hline Clinical Stage & $\begin{array}{c}\text { OR, } 0.94 \\
(95 \% \text { CI, } 0.42-2.10)\end{array}$ & .88 & Clinical stage & $\begin{array}{c}\text { OR, } 0.99 \\
(95 \% \text { CI, } 0.44-2.27)\end{array}$ & .99 \\
\hline Age & $\begin{array}{c}\text { OR, } 0.97 \\
(95 \% \text { CI, } 0.93-1.01)\end{array}$ & .15 & Age & $\begin{array}{c}\text { OR, } 0.98 \\
(95 \% \text { CI, } 0.94-1.02)\end{array}$ & .24 \\
\hline ECOG performance status & $\begin{array}{c}\text { OR, } 1.29 \\
(95 \% \text { CI, } 0.58-2.88)\end{array}$ & .53 & ECOG performance status & $\begin{array}{c}\text { OR, } 1.53 \\
(95 \% \text { CI, } 0.66-3.59)\end{array}$ & .32 \\
\hline Omnibus test of model coefficients & N/A & .03 & Omnibus test of model coefficients & N/A & $<.001$ \\
\hline Hosmer-Lemeshow test & N/A & .64 & Hosmer-Lemeshow test & N/A & .81 \\
\hline Nagelkerke pseudoR $^{2}$ & 0.14 & N/A & Nagelkerke pseudoR ${ }^{2}$ & 0.24 & N/A \\
\hline C-statistic & 0.64 & N/A & C-statistic & 0.70 & N/A \\
\hline
\end{tabular}

Checklist, which is a HRQOL instrument validated in general populations with cancer. ${ }^{11}$ Another potential limitation is that although they performed multivariate analyses, they did not control for many important confounders, such as stage and whether adjuvant therapy was given. Furthermore, they did not control for the type of esophagectomy, which is a potential source of bias in light of the fact that $47 \%$ of patients underwent transhiatal esophagectomy. ${ }^{11}$ In our study, esophagectomies were performed almost exclusively via the transthoracic or 3 -field techniques, and thus we did not control for esophagectomy type. Most recently, Quinten and colleagues $^{15}$ performed a pooled analysis of 30 European randomized controlled trials to determine whether baseline HRQOL was associated with long-term survival. Of the 11 cancer sites included in this pooled analysis, they used data from 1 randomized controlled trial on esophageal cancer that had HRQOL (EORTC QLQ-30) data on 65 patients. ${ }^{15}$ On Cox regression analysis, they found that the physical and social functioning scales were independently associated with overall survival in patients with esophageal cancer. ${ }^{15}$
However, a major limitation of this study is that they did not control for stage or treatment received.

A limitation of many of these studies is that the different components of the HRQOL instrument are considered as separate variables in the multivariate analyses. This can potentially introduce bias in several ways. Most important, this practice separates components of the HRQOL instrument into unique variables that have not been validated on their own but rather as part of the overall HRQOL instrument. It is unclear whether the validated overall HRQOL instrument is itself predictive of survival in these studies. Another potential source of bias is that this practice results in multiplicity of testing; for example, Blazeby and colleagues ${ }^{7}$ input 16 components of the HRQOL instrument into the multivariate model as 16 separate variables. Furthermore, this could lead to another source of bias, which is the use of many more predictors in a model than is conventionally considered acceptable for regression model stability. In general, having less than 5 to 10 events/ outcomes per predictor is considered high risk for model instability. 
In our study, we initially considered only the FACT-E as a unique predictor and did not separate it into the component subscales. In part of an exploratory analysis, we identified that the ECS itself appeared to be a unique predictor. Given that the ECS has not been validated as an independent measure, this result requires further research. A strength of our study is that we controlled for many important confounding variables, including stage and treatment received. Furthermore, we did so using a forced-entry method rather than a stepwise elimination method. This forced-entry method has the effect of controlling simultaneously for all the included predictors and confounders as opposed to stepwise elimination methods that only control for the predictors and confounders that are included in the final model after all the eliminations. The forced-entry method is considered a more robust modeling method when the event rate is high enough to support it. Our models had a sufficiently high event rate with more than 10 events per predictor. Another strength of our study is that we have shown that FACT-E and ECS are associated with survival whether we consider them as continuous, dichotomous, or quartile variables; this mitigates the risk of spurious results that can arise from dichotomizing an inherently continuous variable.

\section{Study Limitations}

Limitations to our study include potential bias because patients who were treated without surgery were staged clinically, whereas those treated surgically were staged pathologically. However, sensitivity analyses using clinical stage for all patients replicated the findings of our primary analyses. In our study, stage did not appear to be independently and significantly associated with survival in this cohort. This likely is due to a lack of power to detect a difference in the effect of stage on survival because of our sample size. We cannot use a study of this size to make commentary on the effect of stage on survival, especially in this study considering mainly stage II and III cases. However, we are able to use that variable (ie, stage) in our multivariable analysis and control for confounding effect of stage as we try to determine the effect of baseline HRQOL on survival. The finding that stage was not associated with survival might also be due to some degree of collinearity between stage and therapy. That being said, our model diagnostics did not reveal a significant degree of collinearity between stage and therapy as they relate to the survival model. Another limitation is that these data were derived from patients enrolled in prospective studies and thus may not be generalizable to patients who are not actively being monitored in a prospective manner.

\section{CONCLUSIONS}

In patients with stage II and III esophageal cancer being considered for chemoradiation therapy, higher baseline
FACT-E and ECS were independently associated with better overall survival. On the basis of a review of the literature, further study is needed to assess the prognostic ability of the baseline HRQOL measures to predict survival using only data available before any treatment. Our findings, using data only available before any treatment, suggest that FACT-E and ECS are independent predictors of 2-year survival and appear to drive the ability of the model to predict 2-year survival. These analyses suggest that ECS increases the predictive/discriminatory power of the model more than FACT-E. Although further study is needed, the ECS may be an even stronger predictor than FACT-E and is a focused, shorter questionnaire than the FACT-E and other esophageal-specific HRQOL instruments. Further studies are needed to assess whether the ECS can be used as a parsimonious prognostic tool to inform patient decision-making and patient selection criteria for studies.

\section{Conflict of Interest Statement}

Authors have nothing to disclose with regard to commercial support.

You can watch a Webcast of this AATS meeting presentation by going to: http://webcast.aats.org/2015/Video/ Tuesday/04-28-15_6C_1520_Kidane.mp4.

The authors thank the Kress Family Chair in Esophageal Cancer for support.

\section{References}

1. Darling GE. Quality of life in patients with esophageal cancer. Thorac Surg Clin. 2013;23:569-75.

2. Safieddine N, Xu W, Quadri SM, Knox JJ, Hornby J, Sulman J, et al. Health-related quality of life in esophageal cancer: effect of neoadjuvant chemoradiotherapy followed by surgical intervention. J Thorac Cardiovasc Surg. 2009; 137:36-42.

3. Gotay CC, Kawamoto CT, Bottomley A, Efficace F. The prognostic significance of patient-reported outcomes in cancer clinical trials. J Clin Oncol. 2008;26: 1355-63.

4. Maisey NR, Norman A, Watson M, Allen MJ, Hill ME, Cunningham D. Baseline quality of life predicts survival in patients with advanced colorectal cancer. Eur J Cancer. 2002;38:1351-7.

5. Djarv T, Lagergren P. Six-month postoperative quality of life predicts long-term survival after oesophageal cancer surgery. Eur J Cancer. 2011;47:530-5.

6. Djarv T, Metcalfe C, Avery KN, Lagergren P, Blazeby JM. Prognostic value of changes in health-related quality of life scores during curative treatment for esophagogastric cancer. J Clin Oncol. 2010;28:1666-70.

7. Blazeby JM, Brookes ST, Alderson D. The prognostic value of quality of life scores during treatment for oesophageal cancer. Gut. 2001;49:227-30.

8. Knox JJ, Wong R, Visbal AL, Horgan AM, Guindi M, Hornby J, et al. Phase 2 trial of preoperative irinotecan plus cisplatin and conformal radiotherapy, followed by surgery for esophageal cancer. Cancer. 2010;116: 4023-32.

9. Darling G, Eton DT, Sulman J, Casson AG, Celia D. Validation of the functional assessment of cancer therapy esophageal cancer subscale. Cancer. 2006;107: 854-63.

10. Mendel DB, Laird AD, Xin X, Louie SG, Christensen JG, Li G, et al. In vivo antitumor activity of SU11248, a novel tyrosine kinase inhibitor targeting vascular endothelial growth factor and platelet-derived growth factor receptors: determination of a pharmacokinetic/pharmacodynamic relationship. Clin Cancer Res. 2003;9:327-37. 
11. van Heijl M, Sprangers MA, de Boer AG, Lagarde SM, Reitsma HB, Busch OR, et al. Preoperative and early postoperative quality of life predict survival in potentially curable patients with esophageal cancer. Ann Surg Oncol. 2010;17: 23-30.

12. Brooks JA, Kesler KA, Johnson CS, Ciaccia D, Brown JW. Prospective analysis of quality of life after surgical resection for esophageal cancer: preliminary results. J Surg Oncol. 2002;81:185-94.

13. Blazeby JM, Conroy T, Hammerlid E, Fayers P, Sezer O, Koller M, et al. Clinical and psychometric validation of an EORTC questionnaire module, the EORTC QLQ-OES18, to assess quality of life in patients with oesophageal cancer. Eur J Cancer. 2003;39:1384-94.

14. Sharma A, Walker LG, Monson JR. Baseline quality of life factors predict long term survival after elective resection for colorectal cancer. Int J Surg Oncol. 2013;2013:269510.

15. Quinten C, Martinelli F, Coens C, Sprangers MA, Ringash J, Gotay C, et al. A global analysis of multitrial data investigating quality of life and symptoms as prognostic factors for survival in different tumor sites. Cancer. 2014;120; 302-11.

Key Words: esophageal cancer, quality of life, survival, predictive model

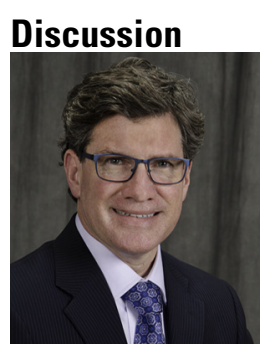

Dr T. Watson (Rochester, NY). I congratulate Dr Kidane and colleagues from the University of Toronto on this nicely presented report assessing the impact of HRQOL on overall survival in patients with esophageal cancer. Staging for cancer can be conceptualized in several ways. Traditionally, anatomic staging has been the standard, whether based on clinical grounds or pathologic interpretation of resected specimens. More recently, the concept of genetic staging has been introduced, with the hope that it ultimately will be more useful and will overcome the limitations inherent to anatomic approaches. What we have heard about today is symptomatic staging of esophageal cancer and how it predicted overall survival in treated patients. The idea of using symptoms, quality of life, or performance status to select patients for therapy is important for us as surgeons as we decide on potentially morbid operations, although we have generally considered the impact on short-term perioperative outcomes rather than on long-term cure. This report today is the first to demonstrate that the severity of preoperative symptoms can be predictive of our ability to cure patients with esophageal cancer.

The concept that more symptomatic patients ultimately do worse is intuitive, although I wonder about confounding variables, particularly tumor stage and treatment type. I was struck by the fact that on univariate analysis, tumor stage did not affect survival, nor did intent of therapy, whether curative or palliative.

I have 3 questions. How do you explain the fact that symptoms appeared more predictive of long-term cure than tumor stage? How was treatment with curative intent defined and why did it not seem to affect overall survival? The differences between mean preoperative FACT-E and
ECS scores between surviving and nonsurviving patients were 5.5 and 6.5 points, respectively. Although these differences were statistically significant, how clinically significant were they and how should we use these data in deciding on treatment recommendations?

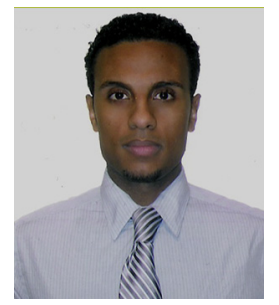

Dr Kidane. For your first question about the symptoms and tumor stage, admittedly, as you said yourself, our methods of staging patients are suboptimal, at best. We believe that the FACT-E and ECS are likely proxies for $\mathrm{T}$ stage. Anecdotally and clinically, we often believe that dysphagia likely represents at least a T3 lesion. We have been looking at our own data to see how FACT-E and ECS correlate with the $\mathrm{T}$ stage, and the short answer, without necessarily getting into it here, is that they do, but not entirely. I think that is where we see the prediction of overall survival. The other thing is that, in terms of staging, esophageal cancer is not the only thing that kills patients with esophageal cancer. It has just been alluded to by Dr Waddell about the patients with lung cancer. Sometimes the treatment that we give them kills them, and sometimes it does it in a way that is paradoxical. Patients who we think have a low enough stage to tolerate our treatments often will get the full vagaries of the treatments we provide and may die sooner than patients with a higher stage.

If you will allow me a little indulgence, in these days of increasingly more complex and expensive staging investigations, it may be a radical reminder that actually something as simple as patient history may be just as valuable. I think the more radical and probably important inference is that something in the patient experience itself may provide some subtle cues about what is going on below the surface, things like a patient's ability to tolerate surgery or maybe some subtle signs of occult metastatic disease, and that may be more valuable than, let's say, an endoscopic ultrasound or a positron emission tomography scan at certain points.

The second question is about the definition of curative intent. Curative intent was a variable we created to distinguish those patients who received definitive chemoradiation therapy as a curative modality, and these would be patients who have squamous cell cancer, from those who received it on a palliative basis. We did not show this, but on Cox regression analysis, curative intent actually was the only other independent predictor of overall survival. We stayed away from presenting those data because we wanted to focus on the use of the Cox regression analyses to elucidate the independent effect of FACT-E and ECS, and all those other variables, although important, were used to adjust and control for their confounding effect, not to look at their own effect themselves. I think the curative intent piece is an interesting piece because it is the physician or clinician side 
of the coin of the FACT-E and ECS. It is somehow a broad gestalt sense that incorporates stage and our estimation of the ability of the person to tolerate the treatment. I think that is one of the reasons why it came out as being the only other independent predictor instead of stage or treatment.

I believe the last question is about whether a mean difference of 5.5 or 6.5 represents a clinically significant difference between ECS or FACT-E. You make an important distinction between clinical and statistical significance, and in the world of patient-reported outcomes, this concept is actually termed the "minimally important difference," and this minimally important difference is defined as the minimal numeric change in a score that results in a meaningful difference to the patient's quality of life. Although there has not been any recent work on the development of such a minimally important difference, in the FACT-E, there has been work in the FACT-G, and that minimally important difference is thought to be approximately 5 . So, going back to our results, a difference of 5.5, I would say it would be safe to potentially extrapolate that that would be a clinically significant or meaningful difference. That being said, the second point I would like to make is that I would take the differences in the mean ECS or mean FACT-E between the 2 groups on univariate analysis with a grain of salt. I say this because that intergroup measure ignores the important intragroup variations in the ECS and FACT-E score itself between each individual patient. It also ignores all the multiple interactions of all the other confounders. So the more valuable way to look at it is through a multivariate logistic or a Cox regression analysis where you can look at each ECS, each FACT-E per individual, and look at how it relates to the different confounders.

In terms of how can we use these data to make treatment recommendations, I don't believe we are at that stage in these data to make too many recommendations. The next steps would be to use these regression data to create or generate receiver operating characteristic curves and then try to identify some sort of cutoffs that would best discriminate between low and high survival groups, and then derive a model that can be externally validated.

Dr Watson. I suggest for the article that you nail down the definitions of curative and palliative, because there can be a lot of overlap. Also think about how we can use these data for meaningful recommendations.

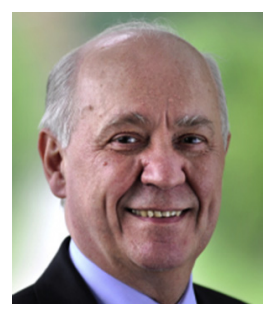

Dr A. Lerut (Leuven, Belgium). I think the group with the low score relates to the FACT-G. The factor that you measure there is poor physical function. Poor physical function relates to more complications after surgery. More complications relate to a higher risk for recurrences. I'm just wondering whether this will be a useful tool to discriminate patients who, for instance, would benefit from trying to upgrade them to a higher score before operation by improving nutritional status, pulmonary function, and so forth. Do you have any ideas on this from your study?

Dr Kidane. I believe Dr Lerut's question is relating to whether prehabilitation can help to augment people's ability to tolerate surgery, and therefore maybe transform their ability to tolerate it. That is an important concept, and I think it is gaining a lot of traction these days. With respect to our study, that is a difficult thing to say, because I believe that FACT-E and ECS encapsulate 2 concepts that we know of. There may be other concepts that we don't understand. One concept is the one you are speaking about, their ability to tolerate surgery, their general functioning, their well-being. The other aspect is about the specific symptoms they have related to cancer, which may not be modifiable by prehabilitation. I think that is an interesting thing that should be looked at going forward.

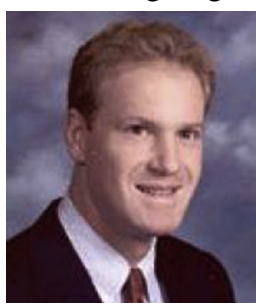

Dr S. DeMeester (Los Angeles, Calif). Very nice study. At face value it makes no sense, but if we look into it a little bit further, maybe there is something to it. Clearly, if you have a T1a lesion, regardless of your quality of life, you are almost certainly going to be cured. If you present with systemic metastases, regardless of your quality of life, you are not likely to survive. So clearly, at the ends of the spectrum, stage matters and quality of life is not as significant an issue. But in the middle, perhaps it's the patient's desire to fight off the lesion. We all know that some of these people are super aggressive about fighting their cancer. They get into health food stores. They change their habits. They do everything they can to fight the tumor. Other people just sort of throw in the towel and wait to die. How much is personal motivation a factor in this? Maybe we can use this in an attempt to bring patients into more involvement with their treatment and care to get a better outcome? Any thoughts on this?

Dr Kidane. That is an interesting viewpoint and comment. I think I'll add to that and say that perhaps the subtle things about the patient's viewpoint about life, about whether they are willing to fight and have this moxie, I guess you could say, may be a reflection of an overall state. Maybe patients with occult metastatic disease don't have that. So there could be 2 points about it, one about the ability of a patient to drive his/her own strength and the other part about whether there is something subtle about the patient experience that medical science hasn't caught up with. 